\title{
Study on collection of the particles in waste gasification gas by wet electrostatic precipitator
}

\author{
Lichun XIAO 1, a * Meng ZHANG Yuchuan WANG \\ ${ }^{1}$ College of Environmental and Chemical Engineering, Yanshan University, Qinhuangdao, China \\ axlc@ysu.edu.cn
}

\begin{abstract}
Keywords: wet electrostatic precipitator; waste gasification gas; collection efficiency; gas discharge;
\end{abstract}

Abstract. Thermal pyrolysis of city life garbage gas is an effective means to achieve waste reduction, harmless treatment and resource utilization. However, the particles in the fuel gas has a detrimental effect on its recycling. It must be purified before recycling. The waste gasification gas purification process by using wet electrostatic precipitator and its feasibility are presented in the paper. The characteristics of waste gasification gas corona discharge are studied under the condition of changing gas pressure, polar matching type and electric field gas speed. Experiment on collection of the dust from a garbage power plant was carried out in a wet electrostatic precipitator. The results show that: waste gasification gas can form a steady corona current in the electrostatic precipitator, and the corona discharge current decreases with the gas pressure. In the main gas components, the increase of $\mathrm{CO}$ content is beneficial to the gas corona discharge while the $\mathrm{CO}_{2}$ is opposite. The corona discharge performance is the best when the polar distance is $300 \mathrm{~mm}$ and the polar matching type is the RS four tooth awn barbed wire to 480C type plate. For the above types of wet electrostatic precipitator, when the water spraying pressure is $0.4 \mathrm{MPa}$, the electric field gas speed is $1.1 \mathrm{~m} / \mathrm{s}$, the collection efficiency of the particles in waste gasification gas will be up to $97.5 \%$. For the municipal solid waste pyrolysis gas which CO component is more than $20 \%$, the wet electrostatic precipitator can be used for purifying its particles effectively.

\section{Introduction}

The annual output of municipal solid waste in China is 1.5 billion tons, and its annual growth rate is up to $8 \% \sim 10 \%$. Realization of reducing and harmless treatment are the most effective method to solve the problem that waste does harm to the ecological environment[1,2]. Thermal pyrolysis and gasification process is one of the most promising technology to recycle the waste. If the pyrolysis gas is used for power generation, its thermal efficiency will be much higher than direct incineration, and it will not produce toxic pollutants such as dioxin[3,4]. The inorganic components in garbage will be converted into glass slag. It can be used as construction materials, this will realize the waste harmless treatment and resource utilization. However, there are a large number of fine particles in the waste gasification gas, which will cause damage to the gas turbine and other power generation equipment and shorten the service life of the gas turbine.

At present, the research on the particle gasification gas purification material is more concentrated in the bag filter technology, but the bag type dust collector equipment has these shortcoming such as high resistance loss and cost, difficult recover and degradation on the replaced bag. It will cause secondary pollution. Wet electrostatic precipitator (ESP) is a fine dust removal equipment with high collection efficiency[5-7]. If it is applied to remove particles from waste gasification gas, it will overcome the shortcomings of the existing bag type dust remover. The feasibility of the wet ESP for purifying the waste gas is studied in this paper. It has opened up a new idea on the application of wet ESP in domestic waste gasification gas purification. 


\section{Experimental principle and equipment}

The basic working principle of wet ESP includes particle charged, charged particle migration and trapping, removing the dust on the surface of collecting plate[8-10]. The details are as follows: (1) The high voltage electric field area in the wet ESP are full of small droplets generating from the centrifugal atomizing nozzle; (2) Then the atomizing droplets will be charged on the condition that corona discharge is produced when the electric field is added an DC high voltage. (3) Free electrons and charged particles will increase greatly under the interaction between the dust and the charged droplets. (4) A layer of continuous flow of water film will be formed on the surface of polar plate in order to wash away the dust attached to the plate[11-13].

Fig. 1 shows the experimental device of the wet ESP. The experimental equipment is mainly composed of a high voltage power supply system, a discharge electrode, a dust collection plate and a water spraying system. The discharge electrode and dust collection system are the core of the wet ESP. The water spraying system can control the flow and pressure of the atomizing nozzle, and the high voltage power supply system provides a DC high voltage power supply for the discharge electrode and the dust collection plate through a high voltage silicon rectifying device.

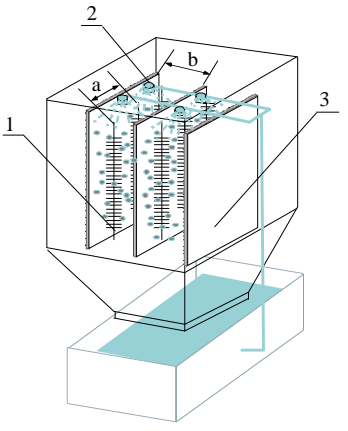

(a)Schematic diagram of the wet ESP

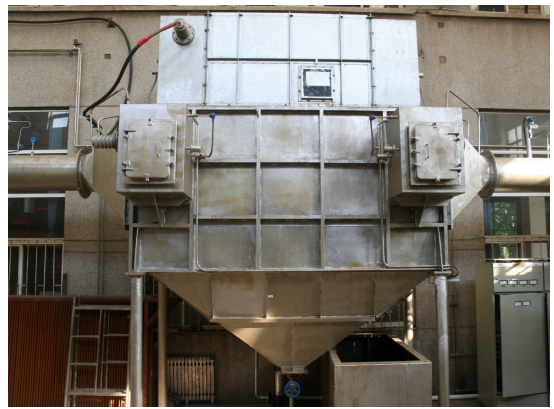

(b) Photo of wet ESP

1. Discharge electrode 2. Water spraying system 3. Dust collection plate

Fig.1 Experimental device of wet ESP

The dust content in the gas was measured by the isokinetic sampling method in the experiment. The sampling system is shown in Fig.2. The dust gas in turn passes through the sampling tube, the drying bottle, the suction pump, the buffer bottle and the flowmeter, and the sampling speed is controlled by the adjusting valve to achieve isokinetic sampling.

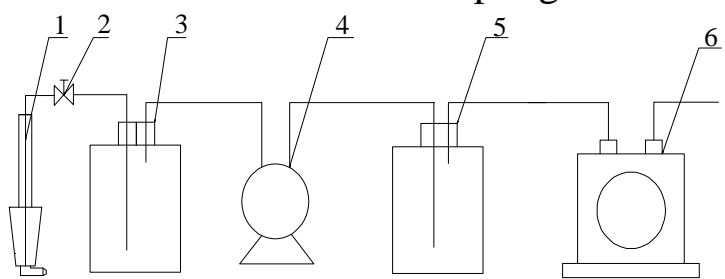

1.sampling tube 2.adjusting valve 3.drying tube 4. suction pump 5. buffer bottle 6 . flowmeter

Fig.2 Dust content measurement device

\section{Results of gas discharge}

\section{characteristics of gas corona discharge affected by the electrode spacing}

As shown in Fig.1, the dimension $a$ is the line spacing of the electrode spacing and $b$ is the plate spacing. The fishbone needling line was used as the discharge electrode and the 480C type plate was used as the anodic plate. The $V-I$ characteristic curve was measured when the dimension a and $\mathrm{b}$ is changed. The experimental results are shown in Fig.3. 


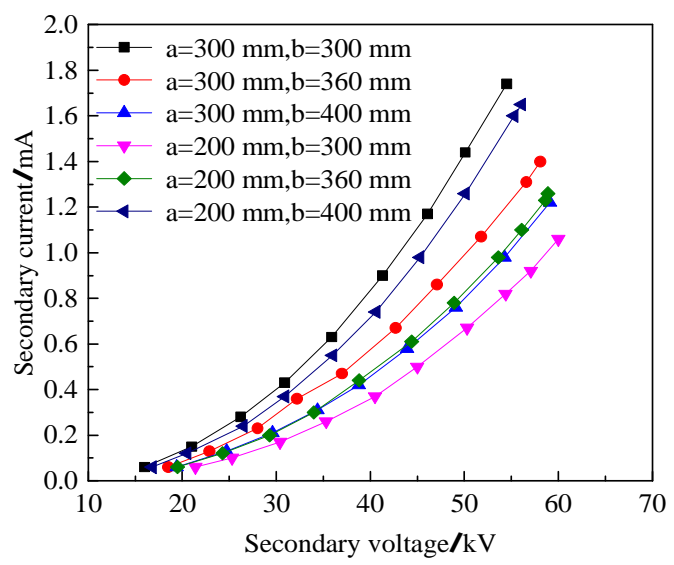

Fig. 3V-I characteristic affected by the electrode spacing

From Fig.3, It can be seen that the $V$ - $I$ characteristic curve produced by the gas corona discharge moves to the right when the distance between the plates increases, i.e., the secondary current decreases with the plate spacing when the secondary voltage is same. The reason is that the electric field intensity between the electrode decreases with the plate spacing. The greater the electric field intensity, the stronger the electron acceleration. The number of electrons generated by corona discharge increases. This will fully increase the secondary current.The secondary current increases with the line spacing when the plate spacing and the secondary voltage is at the same. This is mainly due to the formation of a local electric field around every discharge line. When the line spacing is relatively small, the electric field near the adjacent lines will be weakened due to the combination of the electric field vectors in the boundary area of the power lines. It will results in the decrease of the secondary current. From the experiment, it shows that the performance of gas corona discharge is the best when the line spacing is $300 \mathrm{~mm}$ and the plate spacing is also $300 \mathrm{~mm}$.

\section{characteristics of gas corona discharge affected by the gas pressure}

The fish bone needling cathode line was used as the discharge electrode to measure the $V-I$ characteristic curves. Gas pressure is selected from 7 kPa to $17 \mathrm{kPa}$. As shown in Fig.4,

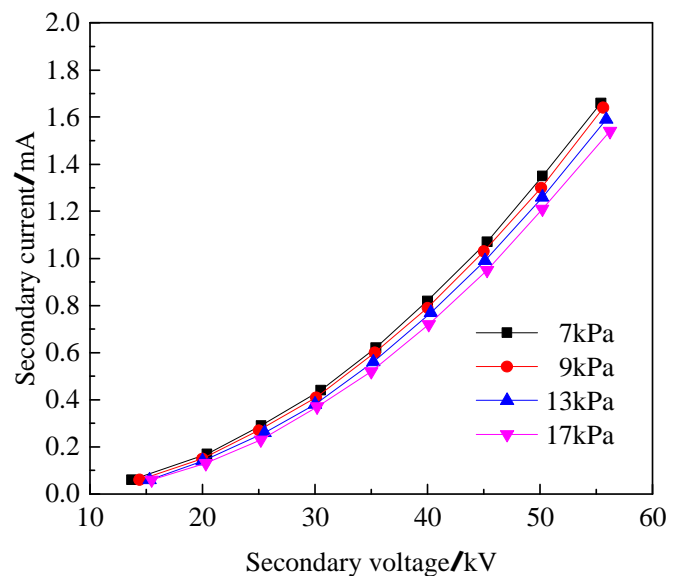

Fig.4 V-I characteristic affected by the gas pressure

From Fig. 4, it can be seen that the $V$ - $I$ characteristic curve moves to the right with the increase of gas pressure. i.e., the greater gas pressure, the smaller secondary current generated by the corona discharge. And the higher gas pressure, the higher corona voltage. The reason is that the corona discharge performance is related to the first ionization coefficient of Townsend $\alpha$ which is directly proportional to the electric field intensity and inversely proportional to the gas pressure according to Townsend discharge theory. When the gas pressure is constant the electric field intensity increases with the secondary voltage. The movement of electrons in the electric field will get higher speed, which will increase the kinetic energy. It can also drive more gas molecules to ionize and produce more free electrons. At last the secondary will increase. When the gas pressure increases, the average free path of particles will be shortened. The energy obtained in the motion of electrons will also be reduced accordingly. So that some electrons do not have enough energy to collide and ionize the gas 
molecules. This will make the number of charged particles reduce and the secondary current decrease. Therefore, the greater the pressure of the gas, the higher the corona inception voltage.

\section{characteristics of gas corona discharge affected by the polar matching type}

Three types of cathode lines which is named fish bone needling line, RS four tooth awn line and BS two tooth prickle line was selected as the discharge electrode in the experiment. The 480C type plate was selected as the collection electrode. When the gas pressure is $7 \mathrm{kPa}$ the $V-I$ characteristic curve of the waste gasification gas is measured. The experimental results are shown in Fig.5.

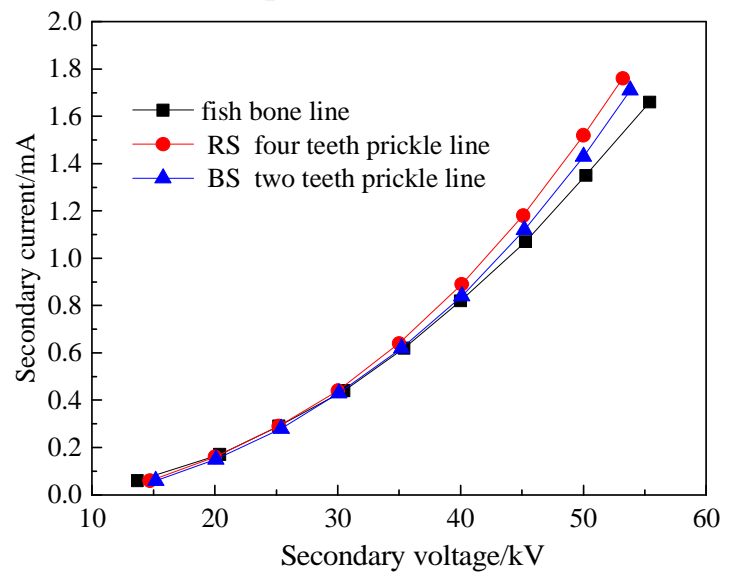

Fig.5 V-I characteristic affected by the polar matching type

As shown in Fig.5, the secondary current generated by the gas discharge increases with the secondary voltage for the three polar matching type. At the same secondary voltage, the secondary current produced by the RS four teeth awn line discharge are the largest and the one of the fish bone needle line are the minimum. It can be seen that the corona discharge performance of the gas produced by the RS four teeth awn line is the best when the working voltage, gas pressure and gas composition are the same. The difference between the spark discharge voltage and the corona discharge voltage is named the effective operation voltage. The larger the effective operation voltage, the better the stability of the wet electrostatic precipitator's operation. From Fig.5, it can be seen that the effective voltage of the three cathode lines are not obvious. The reason is that all kinds of cathode lines will produce obvious corona discharge only on the tip of the discharge line and the ability for the gas ionization is inversely proportional to the tip's radius. For the awn lines, the size of the corona current is determined by the radius and quantity of the tip. The current of RS four teeth awn line is more than that of the BS two teeth awn line because the awn number of the RS four teeth awn line is more. The radius of the fish bone needling line is small, but its awn number is little, so the secondary current produced by the fish bone needling line is less than that by the RS four teeth awn line under the same secondary voltage. Meanwhile the number of discharge for the fish bone needling line is less than the others, its distribution uniformity of current density in the electric field is poor. This will results in its discharge performance is also poor.

\section{characteristics of gas corona discharge affected by the gas component}

The main components of the waste gasification gas from a garbage power plant are as follows: $\mathrm{CO}: 21.20 \%, \mathrm{CO}_{2}: 11.20 \%, \mathrm{H}_{2}: 0.59 \%, \mathrm{CH}_{4}: 0.49 \%, \mathrm{~N}_{2}: 66.21 \%$. It can be seen that the content of $\mathrm{H}_{2}$ and $\mathrm{CH}_{4}$ in the gas is very low, and the main components are $\mathrm{N}_{2}, \mathrm{CO}_{2}$ and $\mathrm{CO}$. The gas pressure was set to $7 \mathrm{kPa}$ and the gas was prepared according to table 1 . The influence of the three gases on the V-I characteristics is studied. The experimental results are shown in Fig.6.

Tab.1 Gas component of each test

\begin{tabular}{cccc}
\hline number & $\mathrm{N}_{2}(\%)$ & $\mathrm{CO}_{2}(\%)$ & $\mathrm{CO}(\%)$ \\
\hline $1 \#$ & $\mathbf{3 0}$ & $\mathbf{7 0}$ & - \\
$2 \#$ & $\mathbf{5 0}$ & $\mathbf{5 0}$ & - \\
$3 \#$ & $\mathbf{7 0}$ & $\mathbf{3 0}$ & - \\
$4 \#$ & $\mathbf{7 0}$ & $\mathbf{1 0}$ & $\mathbf{2 0}$ \\
$5 \#$ & $\mathbf{3 0}$ & - & $\mathbf{5 0}$ \\
$6 \#$ & $\mathbf{5 0}$ & - & $\mathbf{3 0}$ \\
$7 \#$ & $\mathbf{7 0}$ & & \\
\hline
\end{tabular}




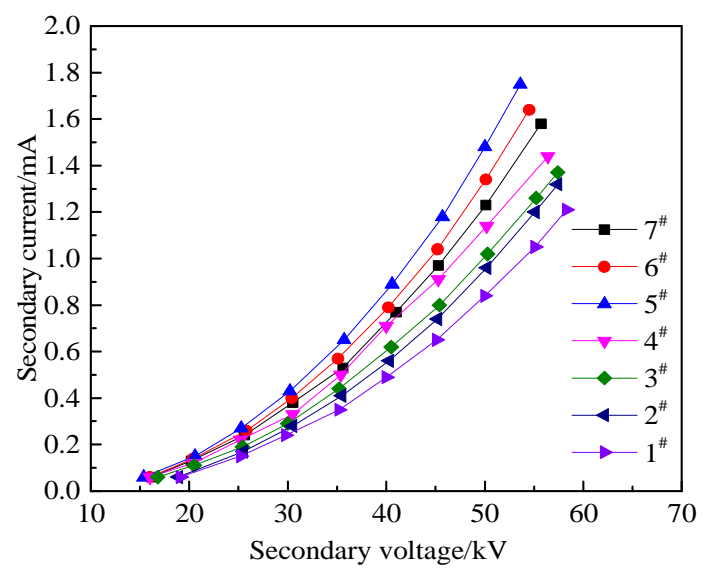

Fig.6 V-I characteristic affected by the gas component

According to the V-I characteristic curves of 1\#, 2\# and 3\# in Fig.6, the V-I characteristic curve gradually shifts to the right when the $\mathrm{CO}_{2}$ content increases, i.e., the more $\mathrm{CO}_{2}$ content, the lower secondary current. According to the $V-I$ characteristic curves of $5 \#, 6 \#$ and 7\#, the secondary current simultaneously increases with the content of $\mathrm{CO}$ when the secondary voltage doesn't change. According to the V-I characteristic curves of 3\#, 4\# and 7\#, the secondary current decrease with the content of $\mathrm{CO}_{2}$ when the content of $\mathrm{N}_{2}$ doesn't change. In short, the increase of $\mathrm{CO}$ content is beneficial to gas corona discharge, while the increase of $\mathrm{CO}_{2}$ content is not conducive to gas corona discharge.The main reason for this phenomenon is that the gas discharge is affected by its electronegativity and ionization energy. $\mathrm{N}_{2}$ has no electronegativity and electronegativity of $\mathrm{CO}$ is extremely low, therefore, the secondary current produced from two kinds of gas mainly depend on their ionization energy. The ionization energy of $\mathrm{CO}$ is low, it can be easily ionized to more free electrons at the same secondary voltage, so its discharge performance becomes better when the content of $\mathrm{CO}$ in the gas increases. However, $\mathrm{CO}_{2}$ is one of the gas which has strong electronegativity, if the content of $\mathrm{CO}_{2}$ increases, on the one hand it will significantly reduce the number of free electrons, this results in the secondary current reduced, on the other hand the diameter and quality of $\mathrm{CO}_{2}$ molecular is more than $\mathrm{CO}$ and $\mathrm{N}_{2}$, and its molecular mean free path is smaller, the energy obtained by accelerated motion is smaller too, the electronic energy is not easy to accumulate in the movement, the probability of collision reduces, this will also result in the secondary current reduced.

\section{Results of dust precipitation}

\section{Collection efficiency affected by the water spraying pressure}

As can be seen from Fig. 7, the collection efficiency increases with the water pressure when the pressure changes from 0.2 $\mathrm{MPa}$ to $0.4 \mathrm{MPa}$. When the water pressure is greater than $0.4 \mathrm{MPa}$, it has little influence on the dust collection efficiency. The reason is that water pressure has great influence on the diameter and number of droplets. When the water pressure is less than $0.4 \mathrm{MPa}$, the diameter of droplets produced by the nozzle is larger, and it decreases with the water pressure. When the water pressure changes to $0.4 \mathrm{MPa}$, the droplets becomes more fine and dense, and it no longer change when the water pressure continues to increase. Therefore, when water pressure increases, the number of droplets will also increase. It will increase the probability of collisions between free electrons and droplets and generate more charged droplets, and increase the probability of interaction between charged droplets and dust particles. This will promote the dust precipitation. When the water pressure is greater than 0.4 $\mathrm{MPa}$, it has little influence on the dust removal efficiency, and water consumption and energy consumption will turn large. Therefore, the appropriate operating water pressure range is 0.35 0.45 MPa, which can not only achieve high dust removal efficiency, but also reduce energy consumption. 


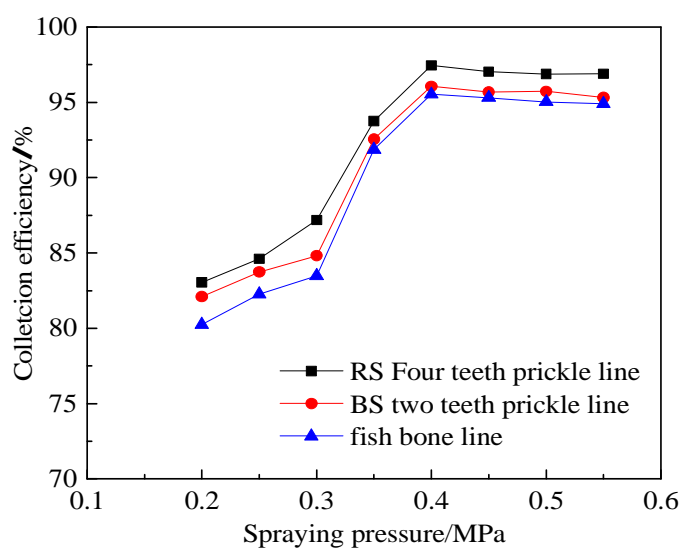

Fig.7 collection efficiency affected by the water pressure

\section{Collection efficiency affected by the electric field gas velocity}

As can be seen from Fig.8, the dust removal efficiency of the wet electrostatic precipitator increases with the electric field gas velocity when it is less than $1.1 \mathrm{~m} / \mathrm{s}$, then the dust removal efficiency decreases with it. This is because the gas velocity has great influence on the dust velocity of convergence. When the gas velocity is low, the dust velocity increases with the gas velocity, but when the gas velocity is greater than a certain value, it will decrease. According to the Deustch formula, the dust removal efficiency is positively related to the dust velocity. Therefore it increases with the gas velocity and then decreases with it. When the electric field gas velocity is too high, it will cause the gas distribution uneven and occurrence of "secondary dust", this will reduce the dust removal efficiency.

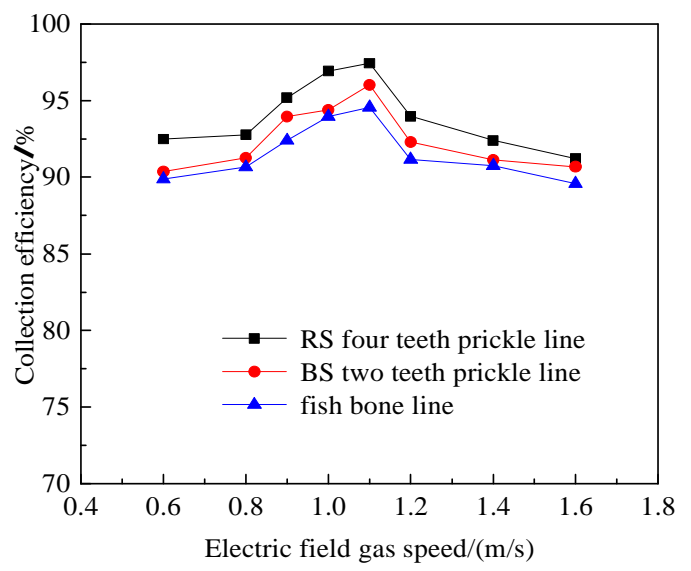

Fig.8 Collection efficiency affected by the electric field gas velocity

\section{Collection efficiency affected by the polar matching type}

The dust removal experiment was made on the following conditions: the electric field gas velocity was $1.1 \mathrm{~m} / \mathrm{s}$, the water pressure was $0.4 \mathrm{MPa}$, and the operating voltage was the spark tracing voltage, the anodic plate was selected as 480C type plate, the results of dry and wet ESP are shown in Table 2.

Tab. 2 Collection efficiency of wet and dry ESP

\section{Polar matching type}

Collection efficiency $(\%)$

Wet ESP Dry ESP

\begin{tabular}{llr} 
BS four teeth awn line & 97.44 & 86.73 \\
RS two teeth awn line & 96.02. & 83.45 \\
Fish bone needle line & 94.95. & 80.66 \\
\hline
\end{tabular}

From table 2, it can be seen that the collection efficiency of the RS four teeth awn line is the highest. It is up to $97.54 \%$. The one of the fish bone needle line is the lowest. This is mainly because the 
secondary current produced by the RS four teeth awn line is the biggest than the others though their spark tracing voltage are almost equal. It will promote more dust particles charged and increase the collection efficiency. The secondary current produced by the fish bone needle line is the lowest, so its dust removal effect is the worst. The collection efficiency of the wet ESP is better than the dry one under three types of electrode. The reason is that there are larges of droplets in the electric field of the wet ESP, which can enhance the coagulation effect of the dust. The collected dust in the wet ESP is not easy to appear "secondary dust" emission. Therefore, the collection efficiency of the wet ESP is better than that of the dry ESP.

\section{Conclusion}

Through the experiment on the discharge of the waste gasification gas and dust precipitation by changing the wet ESP operating parameters, Conclusions are drawn as follows: The waste gasification gas can produce corona discharge in the electrostatic precipitator. It meets the basic operation conditions of the wet electrostatic precipitator which must has a stable corona current. The gas inception voltage increases with the gas pressure. The performance of gas corona discharge is the best when the line spacing is $300 \mathrm{~mm}$ and the plate spacing is also $300 \mathrm{~mm} . \mathrm{CO}$ in the gas has advantage to the corona discharge from the voltage-ample characteristic curve. The best polar matching type for corona discharge is between RS four teeth awn line and 480C type plate. When the electric field gas velocity is $1.1 \mathrm{~m} / \mathrm{s}$, the water pressure is $0.4 \mathrm{MPa}$, and the operating voltage is the spark tracing voltage, the discharge electrode is RS four teeth awn line, the collection efficiency of wet ESP can reach $97.54 \%$. It is $10.67 \%$ higher than the dry ESP.

\section{Acknowledgment}

This research was supported by the Natural Science Foundation of Hebei pvovince, China(E2015203236).

\section{References}

[1] S. Xiao, H. Dong, Y. Geng, M. Brander, An overview of China's recyclable waste recycling and recommendations for integrated solutions, RESOUR CONSERV RECY, Vol.134 (2018), p.112-120.

[2] G. Wang, J. Deng, Z. Ma, Characteristics of filterable and condensable particulate matter emitted from two waste incineration power plants in China., The Science of the total environment, Vol.639 (2018), p.695-704.

[3] Y. Liu, W. Sun, J. Liu, Greenhouse gas emissions from different municipal solid waste management scenarios in China: Based on carbon and energy flow analysis, WASTE MANAGE, Vol. 68 (2017), p. 653-661.

[4] Z. Tao, S. Dai, X. Chai, Mercury emission to the atmosphere from municipal solid waste landfills: A brief review, ATMOS ENVIRON, Vol.170 (2017), p.303-311.

[5] A.K.S. Parihar, T. Hammer, G. Sridhar, Development and testing of plate type wet ESP for removal of particulate matter and tar from producer gas, RENEW ENERG, Vol.77 (2015), p. 473-481.

[6] L. Dey, C. Venkataraman, A Wet Electrostatic Precipitator (WESP) for Soft Nanoparticle Collection, AEROSOL SCI TECH, Vol.46 (2012), p. 750-759.

[7] A. Bologa, H. Paur, M. Lehner, Collection of Fine Particles by Novel Wet Electrostatic Precipitator, IEEE T IND APPL, Vol.45 (2009), p. 2170-2177.

[8] D. Pan, L. Yang, H. Wu, R. Huang, Removal characteristics of sulfuric acid aerosols from coal-fired power plants, J AIR WASTE MANAGE, Vol.67 (2017), p. 352-357.

[9] L. Wang, C. Zheng, Y. Zhang, Speciation Characteristics and Mobility of Trace Elements Across Ultralow Emission Air Pollution Control Devices, ENERG FUEL, Vol. 31 (2017), p. 
13963-13971.

[10] Z. Yang, C. Zheng, X. Zhang, C. Li, Y. Wang, W. Weng, X. Gao, Sulfuric Acid Aerosol Formation and Collection by Corona Discharge in a Wet Electrostatic Precipitator, ENERG FUEL, Vol.31 (2017), p. 8400-8406.

[11] M. Kobayashi, Y. Nakao, Inhibition and elimination of carbon deposition in dry gas desulfurization process under oxy-fuel IGCC derived coal gas environment, FUEL,Vol. 152 (2015),p. 19-28.

[12] Z. Guo, Q. Wang, M. Fang, Simulation of a Lignite-Based Polygeneration System Coproducing Electricity and Tar with Carbon Capture, CHEM ENG TECHNOL, Vol. 38 (2015), p. 463-472.

[13] Q. Du, L. Su, H. Dong, The experimental study of a water-saving wet electrostatic precipitator for removing fine particles, J ELECTROSTAT, Vol. 81 (2016), p. 42-47. 